\title{
STABILIZED GALERKIN FINITE ELEMENT METHODS FOR CONVECTION DOMINATED AND INCOMPRESSIBLE FLOW PROBLEMS
}

\author{
GERT LUBE \\ Mathematics Department, Magdeburg University of Technology \\ PF 4120, D-39016 Magdeburg, Germany
}

\begin{abstract}
In this paper, we analyze a class of stabilized finite element formulations used in computation of (i) second order elliptic boundary value problems (diffusion-convection-reaction model) and (ii) the Navier-Stokes problem (incompressible flow model). These stabilization techniques prevent numerical instabilities that might be generated by dominant convection/reaction terms in (i), (ii) or by inappropriate combinations of velocity/pressure interpolation functions in (ii). Stability and convergence results on non-uniform meshes are given in the whole range from diffusion to convection/reaction dominated situations. In particular, we recover results for the streamline upwind and Galerkin/least-squares methods. Numerical results are presented for low order interpolation functions.
\end{abstract}

1. Introduction. We consider two basic models in fluid mechanics:

(i) second order elliptic boundary value problems (modelling diffusionconvection-reaction problems), and

(ii) Navier-Stokes equations (modelling incompressible flow problems).

Standard Galerkin finite element solutions may suffer from numerical instabilities which are generated by dominant convection (and/or reaction) terms in (i), (ii) or by inappriopriate velocity/pressure interpolation functions in (ii).

In the past decade, Hughes and his co-workers introduced the concept of streamline upwind (SU) and Galerkin/least-squares (GLS) methods for (i) and (ii). The SU-stabilization is achieved by adding to the Galerkin formulation a series of integral terms over each finite element involving the product of the basic equations and the advective operator acting on the test function. In the GLS-approach, least-squares forms of the basic equations are added to the Galer-

1991 Mathematics Subject Classification: Primary 65N30.

The paper is in final form and no version of it will be published elsewhere. 
kin formulation. Basic ideas and results for these methods can be found in $[\mathrm{Hu}]$, [Ja], [FFH], [FS], [Wa].

We present a class of stabilized finite element schemes which involves both methods for diffusion-convection-reaction problems and give existence and error results. Furthermore, we derive design properties for the inherent parameters. In particular, we generalize a result in $[\mathrm{FFH}]$. Numerical results in $2 \mathrm{D}$ and $3 \mathrm{D}$ show the performance of the method (cf. $\S 2$ ).

In $\S 3$ we analyze the GLS-method applied to linearized Navier-Stokes equations and derive error estimates and design properties for the discretization parameters. The method allows for arbitrary $C^{0}$-interpolations of velocity-pressure. We generalize a result in $[\mathrm{DW}],[\mathrm{FS}]$ for the Stokes problem to the non-symmetric case.

The estimates are valid on non-uniform meshes. For the GLS-method they involve control of weighted discrete residuals. Both facts could be exploited in adaptive mesh refinement methods.

Extensions to unsteady problems and more complicated nonlinear problems are possible but not discussed here (cf. [Hu], [HS], [Tea], [Teb], [Ja]).

For $G \subseteq \Omega$ we denote by $W^{k, p}(G)$ the Sobolev space of functions with derivatives of order $\leq k$ belonging to $L^{p}(G)$. The norm and seminorm on $W^{k, p}(G)$ are denoted by $\|\cdot\|_{k, p, G}$ and $|\cdot|_{k, p, G}$, respectively; $(\cdot, \cdot)_{G}$ is the inner product in $L^{2}(G)$.

\section{Stationary diffusion-convection-reaction problems}

2.1. Stabilized Galerkin methods. Let $\Omega \subset \mathbb{R}^{d}, d \leq 3$, be a bounded domain with a Lipschitz continuous boundary $\Gamma=\partial \Omega$. We consider the following second order elliptic boundary value problem modelling steady diffusion-convectionreaction problems:

$$
\begin{aligned}
L u:=-\varepsilon \Delta u+\mathbf{b} \cdot \nabla u+c u & =f & & \text { in } \Omega, \\
u & =0 & & \text { on } \Gamma
\end{aligned}
$$

with the following assumptions:

$$
\begin{aligned}
& \varepsilon>0, \quad \mathbf{b} \in L^{\infty}(\Omega)^{d}, \quad \nabla \cdot \mathbf{b}=0 \quad \text { a.e. in } \Omega, \\
& c \in L^{\infty}(\Omega), \quad c \geq 0 \quad \text { a.e. in } \Omega, \quad f \in L^{2}(\Omega) .
\end{aligned}
$$

A weak solution $u \in V:=W_{0}^{1,2}(\Omega)$ of (2.1), (2.2) satisfies

$$
B_{G}(u, v):=\varepsilon(\nabla u, \nabla v)_{\Omega}+(\mathbf{b} \cdot \nabla u+c u, v)_{\Omega}=(f, v)_{\Omega} \quad \forall v \in V .
$$

Let now $\mathcal{T}_{h}=\{K\}$ be a triangulation of $\bar{\Omega}=\bigcup \bar{K}$ with shape-regular elements $K$ of diameter $h_{K}$. Further, let

$$
V_{h}:=\left\{v_{h} \in V:\left.v_{h}\right|_{K} \in P_{l}(K) \forall K \in \mathcal{T}_{h}\right\},
$$

a usual conforming finite element space of piecewise polynomials of degree $l \geq 1$ 
satisfying an inverse estimate

$$
\exists C_{I}>0: \quad C_{I} \sum_{K} h_{K}^{2}\left\|\Delta v_{h}\right\|_{0,2, K}^{2} \leq\left|v_{h}\right|_{1,2, \Omega}^{2} \quad \forall v_{h} \in V_{h} .
$$

The standard Galerkin finite element solution $u_{h}$ of

$$
u_{h} \in V_{h}: \quad B_{G}\left(u_{h}, v_{h}\right)=\left(f, v_{h}\right)_{\Omega} \quad \forall v_{h} \in V_{h}
$$

may suffer from numerical oscillations that are generated by dominant convective or reaction terms. Under assumption (H.1), a solution $u \in V$ satisfies $L u=f$ in $L^{2}(\Omega)$ and thus with $\psi(v) \in L^{2}(\Omega)$

$$
B_{G}(u, v)+\sum_{K}(L u-f, \psi(v))_{K}=(f, v)_{\Omega} \quad \forall v \in V .
$$

Stabilized Galerkin methods of residual type start from (2.6):

$$
u_{h} \in V_{h}: \quad B_{S G}\left(u_{h}, v_{h}\right)=L_{S G}\left(v_{h}\right) \quad \forall v_{h} \in V_{h}
$$

where

$$
\begin{aligned}
B_{S G}(u, v) & :=B_{G}(u, v)+\sum_{K}(L u, \psi(v))_{K}, \\
L_{S G}(v) & :=(f, v)_{\Omega}+\sum_{K}(f, \psi(v))_{K} .
\end{aligned}
$$

We consider the following class of methods with

$$
\left.\psi\left(v_{h}\right)\right|_{K}:=\delta_{K} \mathbf{b} \cdot \nabla v_{h}+\gamma_{K}\left(-\varepsilon \Delta v_{h}+c v_{h}\right) \quad \forall K \in \mathcal{T}_{h}
$$

and the following (minimal) design properties:

$$
\begin{gathered}
0 \leq \gamma_{K} \leq \delta_{K} \leq \bar{\delta} \leq \frac{1}{2\|c\|_{0, \infty, \Omega}}, \\
\varepsilon \delta_{K} \leq A_{\delta} h_{K}^{2}, \quad A_{\delta}<\frac{1}{2} C_{I} \quad\left(C_{I} \text { from }(2.4)\right) .
\end{gathered}
$$

The properties (H.2) are valid for the following schemes:

(i) $\gamma_{K}=\delta_{K}=0 \quad$ Galerkin finite element $\operatorname{method}(\mathrm{G})$,

(ii) $\gamma_{K}=0, \delta_{K}=0$ streamline upwind finite element method (SU),

(iii) $\gamma_{K}=\delta_{K}>0 \quad$ Galerkin/least-squares finite element method (GLS).

2.2. Auxiliary results. The stabilizing effect of the parameters $\delta_{K}$ and $\gamma_{K}$ can be seen from

Lemma 2.1. For $v_{h} \in V_{h}$ we have under the assumptions (H.1), (H.2)

$$
B_{S G}\left(v_{h}, v_{h}\right) \geq C_{0}\left\|v_{h}\right\|^{2} \quad \text { with } C_{0}=1-\frac{1}{\sqrt{2}}
$$


where

$$
\begin{aligned}
\left.\left\|v_{h}\right\|\right|^{2}:= & \varepsilon\left|v_{h}\right|_{1,2, \Omega}^{2}+\left\|\sqrt{c} v_{h}\right\|_{0,2, \Omega}^{2}+\sum_{K} \delta_{K}\left\|\mathbf{b} \cdot \nabla v_{h}\right\|_{0,2, K}^{2} \\
& +\sum_{K} \gamma_{K}\left\|-\varepsilon \Delta v_{h}+c v_{h}\right\|_{0,2, K}^{2} .
\end{aligned}
$$

Proof. We set $u_{h}=v_{h}$ in (2.7), (2.8) and find that

$$
\begin{aligned}
& B_{G}\left(v_{h}, v_{h}\right)=\varepsilon\left(\nabla v_{h}, \nabla v_{h}\right)_{\Omega}+\left(\left(c-\frac{1}{2} \nabla \cdot \mathbf{b}\right) v_{h}, v_{h}\right)_{\Omega} \quad \text { (integration } \\
& =\varepsilon\left|v_{h}\right|_{1,2, \Omega}^{2}+\left\|\sqrt{c} v_{h}\right\|_{0,2, \Omega}^{2} \\
& \text { by parts) } \\
& \text { (by }(\text { H.1)), } \\
& \text { (2.12) } B_{S G}\left(v_{h}, v_{h}\right)=B_{G}\left(v_{h}, v_{h}\right) \\
& +\sum_{K} \delta_{K}\left\{\left\|\mathbf{b} \cdot \nabla v_{h}\right\|_{0,2, K}^{2}+\left(-\varepsilon \Delta v_{h}+c v_{h}, \mathbf{b} \cdot \nabla v_{h}\right)_{K}\right\} \\
& +\sum_{K} \gamma_{K}\left\{\left\|-\varepsilon \Delta v_{h}+c v_{h}\right\|_{0,2, K}^{2}\right. \\
& \left.+\left(\mathbf{b} \cdot \nabla v_{h},-\varepsilon \Delta v_{h}+c v_{h}\right)_{K}\right\} \\
& \geq\left.||\left|v_{h}\right|\right|^{2}-|\mathrm{I}|-|\mathrm{II}|
\end{aligned}
$$

with

$$
\begin{aligned}
(2.13)|\mathrm{I}|= & \left|\sum_{K} \delta_{K}\left(-\varepsilon \Delta v_{h}+c v_{h}, \mathbf{b} \cdot \nabla v_{h}\right)_{K}\right| \\
\leq & \left(\sum_{K} \varepsilon^{2} \delta_{K}\left\|\Delta v_{h}\right\|_{0,2, K}^{2}\right)^{1 / 2}\left(\sum_{K} \delta_{K}\left\|\mathbf{b} \cdot \nabla v_{h}\right\|_{0,2, K}^{2}\right)^{1 / 2} \\
& +\left(\sum_{K} \delta_{K}\left\|c v_{h}\right\|_{0,2, K}^{2}\right)^{1 / 2}\left(\sum_{K} \delta_{K}\left\|\mathbf{b} \cdot \nabla v_{h}\right\|_{0,2, K}^{2}\right)^{1 / 2} \\
\leq & \frac{1}{2}\left(\alpha_{1}+\alpha_{2}\right) \sum_{K} \delta_{K}\left\|\mathbf{b} \cdot \nabla v_{h}\right\|_{0,2, K}^{2} \\
& +\frac{1}{2 \alpha_{1}} \varepsilon A_{\delta} \sum_{K} h_{K}^{2}\left\|\Delta v_{h}\right\|_{0,2, K}^{2}+\frac{1}{2 \alpha_{2}} \bar{\delta}\|c\|_{0, \infty, \Omega} \sum_{K}\left\|\sqrt{c} v_{h}\right\|_{0,2, K}^{2} \\
\leq & \frac{1}{2}\left(\alpha_{1}+\alpha_{2}\right) \sum_{K} \delta_{K}\left\|\mathbf{b} \cdot \nabla v_{h}\right\|_{0,2, K}^{2}+\frac{1}{4 \alpha_{1}} \varepsilon\left|v_{h}\right|_{1,2, \Omega}^{2} \\
& +\frac{1}{4 \alpha_{2}}\left\|\sqrt{c} v_{h}\right\|_{0,2, \Omega}^{2} \quad(\text { by }(2.4) \text { and }(\mathrm{H} .2)), \\
|\mathrm{II}|= & \left|\sum_{K} \gamma_{K}\left(\mathbf{b} \cdot \nabla v_{h},-\varepsilon \Delta v_{h}+c v_{h}\right)_{K}\right| \\
\leq & \left(\sum_{K} \delta_{K}\left\|\mathbf{b} \cdot \nabla v_{h}\right\|_{0,2, K}^{2}\right)^{1 / 2}\left(\sum_{K} \gamma_{K}^{2} \delta_{K}^{-1}\left\|-\varepsilon \Delta v_{h}+c v_{h}\right\|_{0,2, K}^{2}\right)^{1 / 2}
\end{aligned}
$$


$\leq \frac{1}{2} \alpha_{3} \sum_{K} \delta_{K}\left\|\mathbf{b} \cdot \nabla v_{h}\right\|_{0,2, K}^{2}+\frac{1}{2 \alpha_{3}} \sum_{K} \gamma_{K}\left\|-\varepsilon \Delta v_{h}+c v_{h}\right\|_{0,2, K}^{2} \quad($ by $(\mathrm{H} .2))$.

From (2.12)-(2.14) it follows that

$$
\begin{aligned}
B_{S G}\left(v_{h}, v_{h}\right) \geq & \left\|\left|v_{h}\right|\right\|^{2}-\frac{1}{4 \alpha_{1}} \varepsilon\left|v_{h}\right|_{1,2, \Omega}^{2}-\frac{1}{4 \alpha_{2}}\left\|\sqrt{c} v_{h}\right\|_{0,2, \Omega}^{2} \\
& -\frac{1}{2}\left(\alpha_{1}+\alpha_{2}+\alpha_{3}\right) \sum_{K} \delta_{K}\left\|\mathbf{b} \cdot \nabla v_{h}\right\|_{0,2, K}^{2} \\
& -\frac{1}{2 \alpha_{3}} \sum_{K} \gamma_{K}\left\|-\varepsilon \Delta v_{h}+c v_{h}\right\|_{0,2, K}^{2}
\end{aligned}
$$

and with $\alpha=\alpha_{1}=\alpha_{2}=\frac{1}{2} \alpha_{3}=1 /(2 \sqrt{2})$,

$$
B_{S G}\left(v_{h}, v_{h}\right) \geq\left(1-\frac{1}{\sqrt{2}}\right)\left\|v_{h}\right\|^{2} \text {. }
$$

R e mark 2.1. For the Galerkin/least-squares method (GLS) with $\gamma_{K}=\delta_{K}>$ 0 we find that

$$
B_{G L S}\left(v_{h}, v_{h}\right) \geq \varepsilon\left|v_{h}\right|_{1,2, \Omega}^{2}+\left\|\sqrt{c} v_{h}\right\|_{0,2, \Omega}^{2}+\sum_{K} \delta_{K}\left\|L v_{h}\right\|_{0,2, K}^{2} .
$$

Furthermore, we need a continuity estimate for $B_{S G}(\cdot, \cdot)$.

Lemma 2.2. For $u, v \in V$ with $\Delta u, \Delta v \in L^{2}(K), \forall K \in \mathcal{T}_{h}$ we have under the assumptions (H.1), (H.2)

$$
\begin{aligned}
\left|B_{S G}(u, v)\right| \leq & \frac{1}{2} C_{0}\|\| v \|\left.\right|^{2}+\frac{1}{C_{0}}\left\{\varepsilon|u|_{1,2, \Omega}^{2}+\|\sqrt{c} u\|_{0,2, \Omega}^{2}\right. \\
& +4 \sum_{K} \delta_{K}\|\mathbf{b} \cdot \nabla u\|_{0,2, K}^{2}+8 \varepsilon A_{\delta} \sum_{K} h_{K}^{2}\|\Delta u\|_{0,2, K}^{2} \\
& \left.+8 \sum_{K} \delta_{K}\|c u\|_{0,2, K}^{2}+\sum_{K} \min \left\{\delta_{K}^{-1} ; \varepsilon^{-1}\|\mathbf{b}\|_{0, \infty, K}^{2}\right\}\|u\|_{0,2, K}^{2}\right\} .
\end{aligned}
$$

Proof. We have

$$
\left|B_{S G}(u, v)\right| \leq|\mathrm{III}|+|\mathrm{IV}|+|\mathrm{V}|
$$

with

$$
\begin{aligned}
|\mathrm{III}| & =\left|B_{G}(u, v)\right| \\
= & \left|\varepsilon(\nabla u, \nabla v)_{\Omega}+(\sqrt{c} u, \sqrt{c} v)_{\Omega}-(u, \mathbf{b} \cdot \nabla v)_{\Omega}\right| \text { (integration by parts) } \\
\leq & \varepsilon|u|_{1,2, \Omega}|v|_{1,2, \Omega}+\|\sqrt{c} u\|_{0,2, \Omega}\|\sqrt{c} v\|_{0,2, \Omega} \\
& +\left\{\begin{array}{ll}
\left(\sum_{K} \delta_{K}^{-1}\|u\|_{0,2, K}^{2}\right)^{1 / 2}\left(\sum_{K} \delta_{K}\|\mathbf{b} \cdot \nabla v\|_{0,2, K}^{2}\right)^{1 / 2} & \text { if } \delta_{K}>0 \\
\left(\varepsilon^{-1} d \sum_{K}\|\mathbf{b}\|_{0, \infty, K}^{2}\|u\|_{0,2, K}^{2}\right)^{1 / 2}\left(\varepsilon \sum_{K}|v|_{1,2, K}^{2}\right)^{1 / 2} & \text { otherwise }
\end{array}\right\}
\end{aligned}
$$




$$
\begin{aligned}
\leq & \frac{1}{4} C_{0}\left(\varepsilon|v|_{1,2, \Omega}^{2}+\|\sqrt{c} v\|_{0,2, \Omega}^{2}\right)+\left\{\begin{array}{ll}
\sum_{K} \delta_{K}\|\mathbf{b} \cdot \nabla v\|_{0,2, K}^{2} & \text { if } \delta_{K}>0 \\
\varepsilon|v|_{1,2, \Omega}^{2} & \text { otherwise }
\end{array}\right\} \\
& +\frac{1}{C_{0}}\left(\varepsilon|u|_{1,2, \Omega}^{2}+\|\sqrt{c} u\|_{0,2, \Omega}^{2}\right. \\
& \left.+\sum_{K} \min \left\{\delta_{K}^{-1} ; \varepsilon^{-1} d\|\mathbf{b}\|_{0, \infty, K}^{2}\right\}\|u\|_{0,2, K}^{2}\right),
\end{aligned}
$$

$$
\begin{aligned}
|\mathrm{IV}|= & \left|\sum_{K} \delta_{K}\left\{(-\varepsilon \Delta u+c u, \mathbf{b} \cdot \nabla v)_{K}+(\mathbf{b} \cdot \nabla u, \mathbf{b} \cdot \nabla v)_{K}\right\}\right| \\
\leq & \frac{1}{2}\left(L_{1}+L_{2}\right) \sum_{K} \delta_{K}\|\mathbf{b} \cdot \nabla v\|_{0,2, K}^{2}+\frac{1}{2 L_{1}} \sum_{K} \delta_{K}\|\mathbf{b} \cdot \nabla u\|_{0,2, K}^{2} \\
& +\frac{1}{2 L_{2}} \sum_{K} \delta_{K}\|-\varepsilon \Delta u+c u\|_{0,2, K}^{2}
\end{aligned}
$$

$$
\begin{aligned}
|\mathrm{V}|= & \left|\sum_{K} \gamma_{K}\left\{(-\varepsilon \Delta u+c u,-\varepsilon \Delta v+c v)_{K}+(\mathbf{b} \cdot \nabla u,-\varepsilon \Delta v+c v)_{K}\right\}\right| \\
\leq & \frac{1}{2}\left(L_{3}+L_{4}\right) \sum_{K} \gamma_{K}\|-\varepsilon \Delta v+c v\|_{0,2, K}^{2} \\
& +\frac{1}{2 L_{3}} \sum_{K} \gamma_{K}\|-\varepsilon \Delta u+c u\|_{0,2, K}^{2}+\frac{1}{2 L_{4}} \sum_{K} \gamma_{K}\|\mathbf{b} \cdot \nabla u\|_{0,2, K}^{2} .
\end{aligned}
$$

We summarize (2.16)-(2.19) with $L_{1}=L_{2}=L_{3}=L_{4}=\frac{1}{4} C_{0}$ :

$$
\begin{aligned}
\left|B_{S G}(u, v)\right| \leq & \frac{1}{2} C_{0}\left\{\varepsilon|v|_{1,2, \Omega}^{2}+\|\sqrt{c} v\|_{0,2, \Omega}^{2}\right. \\
& \left.+\sum_{K} \delta_{K}\|\mathbf{b} \cdot \nabla v\|_{0,2, K}^{2}+\sum_{K} \gamma_{K}\|-\varepsilon \Delta v+c v\|_{0,2, K}^{2}\right\} \\
& +\frac{1}{C_{0}}\left\{\varepsilon|u|_{1,2, \Omega}^{2}+\|\sqrt{c} u\|_{0,2, \Omega}^{2}+4 \sum_{K} \delta_{K}\|\mathbf{b} \cdot \nabla u\|_{0,2, K}^{2}\right. \\
& +8 \sum_{K} \varepsilon^{2} \delta_{K}\|\Delta u\|_{0,2, K}^{2}+8 \sum_{K} \delta_{K}\|c u\|_{0,2, K}^{2} \\
& \left.+\sum_{K} \min \left\{\delta_{K}^{-1} ; \varepsilon^{-1} d\|\mathbf{b}\|_{0, \infty, K}^{2}\right\}\|u\|_{0,2, K}^{2}\right\}
\end{aligned}
$$

which yields (2.15).

2.3. Error analysis and parameter design. We may now state the following convergence result. 
THEOREM 2.3. Under the assumptions (H.1), (H.2), there exists a unique solution $u_{h} \in V_{h}$ of (2.7), (2.8) which converges to the solution of (2.3) as follows:

$$
\begin{aligned}
\left\|u-u_{h} \mid\right\|^{2}:= & \varepsilon\left|u-u_{h}\right|_{1,2, \Omega}^{2}+\left\|\sqrt{c}\left(u-u_{h}\right)\right\|_{0,2, \Omega}^{2} \\
& +\sum_{K} \delta_{K}\left\|\mathbf{b} \cdot \nabla\left(u-u_{h}\right)\right\|_{0,2, K}^{2} \\
& +\sum_{K} \gamma_{K}\left\|-\varepsilon \Delta\left(u-u_{h}\right)+c\left(u-u_{h}\right)\right\|_{0,2, K}^{2} \\
\leq & C \sum_{K} h_{K}^{2 l} B_{K}\left(\varepsilon, h_{K}, \delta_{K}\right)|u|_{l+1,2, K}^{2}
\end{aligned}
$$

if $u \in V \cap W^{l+1,2}(\Omega), l \geq 1$. Furthermore,

$$
\begin{aligned}
B_{K}\left(\varepsilon, h_{K}, \delta_{K}\right):= & \varepsilon+\delta_{K}\|\mathbf{b}\|_{0, \infty, K}^{2} \\
& +\min \left\{\delta_{K}^{-1} ; \varepsilon^{-1}\|\mathbf{b}\|_{0, \infty, K}^{2}\right\} h_{K}^{2}+\|c\|_{0, \infty, K} h_{K}^{2} .
\end{aligned}
$$

P r o of. The existence and uniqueness of $u_{h} \in V_{h}$ are a consequence of Lemmas 2.1, 2.2 and Lax-Milgram's lemma.

Let $e_{h}:=u_{h}-u=\left(u_{h}-\pi_{h} u\right)+\left(\pi_{h} u-u\right) \equiv \vartheta_{h}+\eta_{h}$ where $\pi_{h}: V \rightarrow V_{h}$ denotes the interpolation operator in $V_{h}$. Then

$$
\begin{aligned}
C_{0}\left\|\vartheta_{h}\right\| \|^{2} \leq & B_{S G}\left(\vartheta_{h}, \vartheta_{h}\right) \\
= & B_{S G}\left(e_{h}-\eta_{h}, \vartheta_{h}\right)=-B_{S G}\left(\eta_{h}, \vartheta_{h}\right) \quad \text { (consistency, by (2.6)) } \\
\leq & \frac{1}{2} C_{0}\left\|\left|\vartheta_{h} \|\right|^{2}+\frac{1}{C_{0}}\left\{\varepsilon\left|\eta_{h}\right|_{1,2, \Omega}^{2}+\left\|\sqrt{c} \eta_{h}\right\|_{0,2, \Omega}^{2}\right.\right. \\
& +4 \sum_{K} \delta_{K}\left\|\mathbf{b} \cdot \nabla \eta_{h}\right\|_{0,2, K}^{2}+8 \varepsilon A_{\delta} \sum_{K} h_{K}^{2}\left\|\Delta \eta_{h}\right\|_{0,2, K}^{2} \\
& +8 \sum_{K} \delta_{K}\left\|c \eta_{h}\right\|_{0,2, K}^{2} \\
& \left.+\sum_{K} \min \left\{\delta_{K}^{-1} ; \varepsilon^{-1}\|\mathbf{b}\|_{0, \infty, K}^{2}\right\}\left\|\eta_{h}\right\|_{0,2, K}^{2}\right\} .
\end{aligned}
$$

The estimate (2.22), together with the standard approximation result

implies

$$
\begin{aligned}
\left\|\eta_{h}\right\|_{m, 2, K} \leq C_{A} h_{K}^{l+1-m}|u|_{l+1,2, K} \\
\forall u \in W^{l+1,2}(K), 0 \leq m \leq l+1, \forall K \in \mathcal{T}_{h},
\end{aligned}
$$

$$
\begin{aligned}
\left\|\vartheta_{h}\right\|^{2} \leq & \frac{\sqrt{2}}{C_{0}} C_{A} \sum_{K} h_{K}^{2 l}\left\{\varepsilon+\|c\|_{0, \infty, K} h_{K}^{2}+\delta_{K}\|\mathbf{b}\|_{0, \infty, K}^{2}\right. \\
& \left.+\delta_{K}\|c\|_{0, \infty, K}^{2} h_{K}^{2}+\min \left\{\delta_{K}^{-1} ; \varepsilon^{-1}\|\mathbf{b}\|_{0, \infty, K}^{2} h_{K}^{2}\right\}\right\}|u|_{l+1,2, K}^{2} \\
\leq & C \sum_{K} h_{K}^{2 l} B_{K}|u|_{l+1,2, K}^{2} .
\end{aligned}
$$

Note that by (H.2), $\delta_{K}\|c\|_{0, \infty, K} \leq \frac{1}{2}$. 
We now derive an additional design condition for $\delta_{K}$ by optimizing (2.21), more precisely by balancing the terms

$$
\delta_{K}\|\mathbf{b}\|_{0, \infty, K}^{2} \sim \min \left\{\delta_{K}^{-1} ; \varepsilon^{-1}\|\mathbf{b}\|_{0, \infty, K}^{2}\right\} h_{K}^{2} .
$$

A simple analysis yields with the local Peclet number $\mathrm{Pe}_{K}:=\varepsilon^{-1} h_{K}\|\mathbf{b}\|_{0, \infty, K}$

$$
\begin{aligned}
& \delta_{K} \sim h_{K}\left(\|\mathbf{b}\|_{0, \infty, K}\right)^{-1} \quad \text { if } \mathrm{Pe}_{K} \geq 1, \\
& \delta_{K} \sim h_{K}^{2} \varepsilon^{-1} \quad \text { if } \mathrm{Pe}_{K} \leq 1 \text {. }
\end{aligned}
$$

Then it follows that

$$
B_{K}\left(\varepsilon, h_{K}, \delta_{K}\right) \sim \varepsilon+\|\mathbf{b}\|_{0, \infty, K} h_{K}+\|c\|_{0, \infty, K} h_{K}^{2} .
$$

Corollary 2.4. Under the assumptions (H.1) and (H.2) a, b, c, we have the convergence result

$$
\left.\left|\| u-u_{h}\right|\right|^{2} \leq C \sum_{K} h_{K}^{2 l}\left(\varepsilon+\|\mathbf{b}\|_{0, \infty, K} h_{K}+\|c\|_{0, \infty, K} h_{K}^{2}\right)|u|_{l+1,2, K}^{2} .
$$

R e mark 2.2. For $d=1, c=0, b, f=$ const and $h=h_{K}$, one has a nodally exact solution of the SU- or GLS-method provided that

$$
\delta=\delta_{K}=\frac{h}{2|b|}\left\{\operatorname{coth} \frac{\mathrm{Pe}_{K}}{2}-\frac{2}{\mathrm{Pe}_{K}}\right\},
$$

which is in accordance with the "double-asymptotic" law (2.25) for $\mathrm{Pe}_{K} \gg 1$ and $\mathrm{Pe}_{K} \ll 1$. Unfortunately, such a superconvergence result is not available in multiple dimensions for variable coefficients. Nevertheless, a "double-asymptotic" law of type (2.25) is frequently used in computations [FFH], [Tea], [Teb]. Note that (2.25) has been derived by a local $L^{2}$-error analysis argument only. On the other hand, the SU- and GLS-methods with (2.25) cannot "model" characteristic interior and/or boundary layer (cf. §2.4) and they result in mild oscillations which are restricted to a small neighbourhood of the layers. Based on a refined local error analysis, one can prove that characteristic numerical layers have a "spread" of $O\left(\sqrt{\mathscr{C}} \ln \mathscr{C}^{-1}\right), \mathfrak{a}:=\max \{h ; \varepsilon\}$ [Wa]. For a modified SU-scheme, an $L^{\infty}$-analysis yields even a "spread" of $O\left(h^{3 / 4} \ln h^{-1}\right)$ if $\varepsilon \leq h^{3 / 2}$ for $d=2$ and $\mathbf{b}=(1,0)^{\mathrm{T}}$.

Remark 2.3. The error estimate (2.26) is uniformly valid for $0 \leq \gamma_{K}$ $\leq \delta_{K}$ and thus holds for the SU- and GLS-methods with $\gamma_{K}=0$ and $\gamma_{K}=\delta_{K}$, respectively. For the latter method $\left(2.9^{*}\right)$ holds and there exists additional control of a weighted residual according to

$$
\sum_{K} \delta_{K}\left\|L u_{h}-f\right\|_{0,2, K}^{2} \leq C \sum_{K} h_{K}^{2 l} B_{K}|u|_{l+1,2, K}^{2}
$$

which could be exploited in adaptive methods [Jo].

R e m a r k 2.4. The SU-method does not take into account reaction dominated situations with $\|c\|_{0, \infty, K} h_{K}^{2} \gg \varepsilon$. So it could be desirable to separate more clearly the influence of the convection and reaction terms with $\gamma_{K}>0$. An alternative 
choice to the GLS-method with $\gamma_{K}=\delta_{K}$ is

$$
\begin{aligned}
& \gamma_{K} \sim h_{K}\left(\|\mathbf{b}\|_{0, \infty, K}\right)^{-1} \quad \text { if } \widetilde{\mathrm{Pe}}_{K}:=h_{K}\|\mathbf{b}\|_{0, \infty, \Omega} \varepsilon^{-1} \geq 1, \\
& \gamma_{K} \sim h_{K}^{2} \varepsilon^{-1} \quad \text { if } \widetilde{\mathrm{Pe}}_{K} \leq 1
\end{aligned}
$$

(cf. $\S 3,[\mathrm{Teb}])$.

2.4. Numerical results and extensions. Now we present numerical results for P1 interpolation $(l=1)$ in (2.7), (2.8) for two- and three-dimensional problems using the package GLSFEM written by D. Weiß [W]. Based on the estimates of $\S 2.3$, the calculations were performed with $\gamma_{K}=\delta_{K}$ and

$$
\delta_{K}=\delta^{*} \frac{h_{K}}{2\|\mathbf{b}\|_{0, \infty, K}} \min \left\{1 ; \frac{1}{6} \mathrm{Pe}_{K}\right\}, \quad \mathrm{Pe}_{K}=\frac{h_{K}\|\mathbf{b}\|_{0, \infty, K}}{\varepsilon},
$$

which is in accordance with (2.25). For $\delta^{*} \rightarrow 0$ we recover the Galerkin method.

We denote by $\nu$ the outward unit normal vector on $\Gamma$ and by $\Gamma_{-}, \Gamma_{0}, \Gamma_{+}$the "inflow", "characteristic" and "outflow" parts of the boundary $\Gamma$ where $\mathbf{b} \cdot \nu<0$, $\mathbf{b} \cdot \nu=0$ and $\mathbf{b} \cdot \nu>0$, respectively.

The first examples are devoted to convection dominated problems.

ExAmple 2.1. Let $\Omega=(0,1)^{2} \subset \mathbb{R}^{2}, \varepsilon=10^{-6}, \mathbf{b}=(1 ; 0.5)^{\mathrm{T}}, c=f=0$. A discontinuous profile given at the "inflow" part $\Gamma_{-}$is transported along the streamlines. The solution admits an interior "characteristic" layer at $S$ and an "ordinary" boundary layer at the upper part of the "outflow" boundary $\Gamma_{+}$(cf. Fig. 2.1a). Level lines of the discrete solution of (2.7), (2.8), (2.28) with $\delta^{*}=2$ on an equidistant $20 \times 20$-mesh are given in Fig. 2.1c. The layers are sharply resolved but mild oscillations appear in a small neighbourhood of the layers. For $\delta^{*} \rightarrow 0$ we arrive at the Galerkin solution with global pollution of the oscillations (not shown here). If $\delta^{*}$ is too big, the layers are smeared out (not shown here).

In Fig. $2.1 \mathrm{~b}$ we present the dependence of the discrete $L^{2}$-error (on an equidistant $20 \times 20$-mesh) on the parameter $\delta^{*}$. The error is minimized for $\delta^{*} \sim 2$.

We remark that the local oscillations cannot be avoided with grid refinement (cf. Fig. 2.1d with $\delta^{*}=2$ on an equidistant $100 \times 100$-mesh) and in adaptive codes $[\mathrm{KR}]$.

Discrete solutions with local oscillations appearing in the neighbourhood of layers (cf. Example 2.1) are, in some sense, not satisfactory. As a remedy, [Jb] proposed to introduce an additional term of artificial diffusion depending on the discrete residual, the so-called "shock-capturing streamline upwind method". It reads

$$
u_{h} \in V_{h}: \quad B_{S G}\left(u_{h}, v_{h}\right)+\sum_{K}\left(\beta_{K}\left(u_{h}\right) \nabla u_{h}, \nabla v_{h}\right)_{K}=L_{S G}\left(v_{h}\right)
$$

with

$$
\beta_{K}\left(u_{h}\right):=\beta^{*} h_{K}^{\gamma}\left|\left(L u_{h}-f\right)\right|_{K} \mid, \quad \gamma \in(1.5 ; 2] .
$$




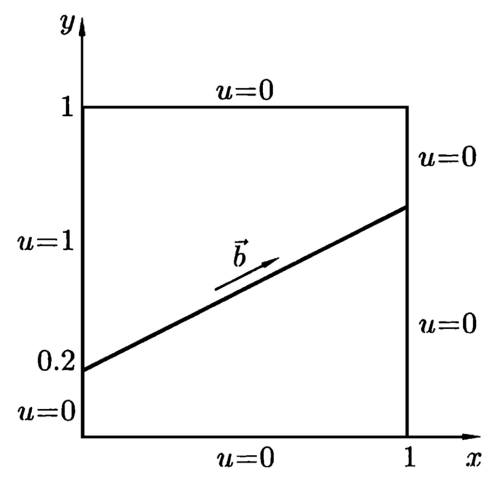

Fig. 2.1a

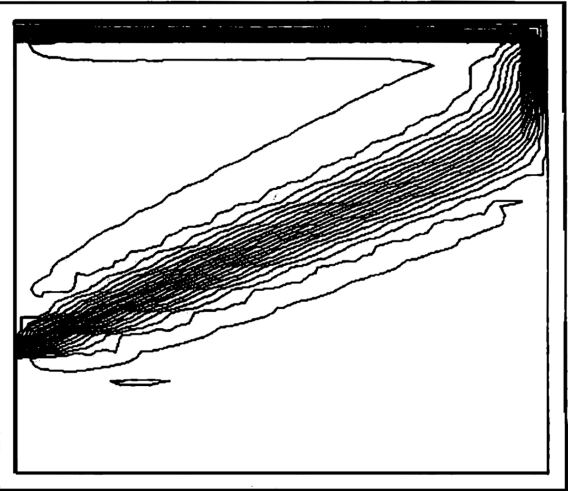

Fig. 2.1c

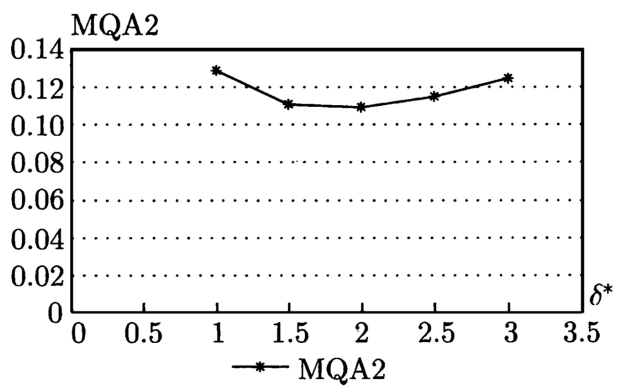

Fig. 2.1b

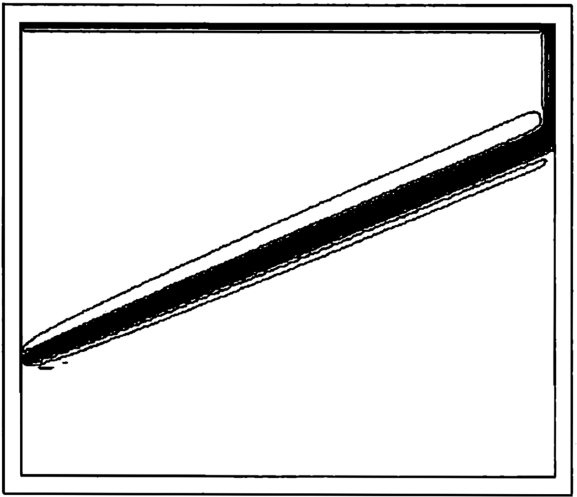

Fig. $2.1 \mathrm{~d}$

One can solve (2.29) by means of simple iteration:

$$
\begin{aligned}
u_{h}^{n+1} \in V_{h}: \quad B_{S G}\left(u_{h}^{n+1}, v_{h}\right)+\sum_{K}\left(\beta_{K}\left(u_{h}^{n}\right) \nabla u_{h}^{n+1}, \nabla v_{h}\right)_{K} & \\
= & L_{S G}\left(v_{h}\right), \quad n=0,1, \ldots
\end{aligned}
$$

EXAMPLE 2.2. We discuss the effect of the "shock-capturing" modification for Example 2.1 using an equidistant $32 \times 32$-mesh and $\delta^{*}=2$. In Table 2.1 we present for different values of $\beta^{*}$ the discrete $L^{2}$-error (MQA) and maximal and minimal values $u_{h}^{\max }$ and $u_{h}^{\min }$, respectively, of $u_{h}$. Note that $0 \leq u \leq 1$ such that $u_{h}^{\max }$ and $u_{h}^{\min }$ represent the effect of numerical oscillations.

Table 2.1. "Shock-capturing streamline upwind method" for Example 2.1 - discrete $L^{2}$-error, $u_{h}^{\max }, u_{h}^{\min }$

\begin{tabular}{|c|c|c|c|}
\hline$\beta^{*}$ & $\mathrm{MQA}=\left\|u-u_{h}\right\|_{0,2, \Omega_{h}}$ & $u_{h}^{\max }$ & $u_{h}^{\min }$ \\
\hline 0.00 & 0.0930 & 1.060 & -0.1037 \\
0.10 & 0.1115 & 1.043 & -0.0533 \\
0.15 & 0.1209 & 1.041 & -0.0351 \\
0.20 & 0.1297 & 1.040 & -0.0199 \\
\hline
\end{tabular}


The oscillations are obviously reduced with increasing $\beta^{*}$ but the solution is smeared out so that the discrete $L^{2}$-error increases with $\beta^{*}$. The discrete solution of (2.29), (2.30) tends to smear with increasing number of iterations in (2.30). The analysis of the scheme (2.29) seems to be open.

In three-dimensional problems we find similar effects of stabilized Galerkin methods as for $d=2$.

EXAmPle 2.3. Let $\Omega=(0,1)^{3} \subset \mathbb{R}^{3}, \varepsilon=10^{-6}, \mathbf{b}=\left(-x_{2}, x_{1}, 0\right)^{\mathrm{T}}, c=f$ $=0$. A discontinuous profile given at the "inflow" part $\Gamma_{-}$is transported along the (curved) streamlines (cf. Fig. 2.2a). At the outflow part $\Gamma_{+}$we impose a homogeneous Neumann condition thus avoiding an "ordinary" boundary layer in the leading term of the asymptotic expansion of the original problem. In Fig. 2.2c we present the outlet profile of the discrete solution (using an equidistant $16 \times 16 \times$ 16-mesh with $\delta^{*}=2$ ) which has again local oscillations in the neighbourhood of the discontinuities of $u$. The dependence of the discrete $L^{2}$-error MQA and $u_{h}^{\max }$, $u_{h}^{\mathrm{min}}$ on the parameter $\delta^{*}$ (on a $16 \times 16 \times 16$-mesh) is given in Fig. 2.2b. The solution is robust with respect to $\delta^{*}$ due to the vanishing "ordinary" boundary layer at the outflow boundary $\Gamma_{+}$.

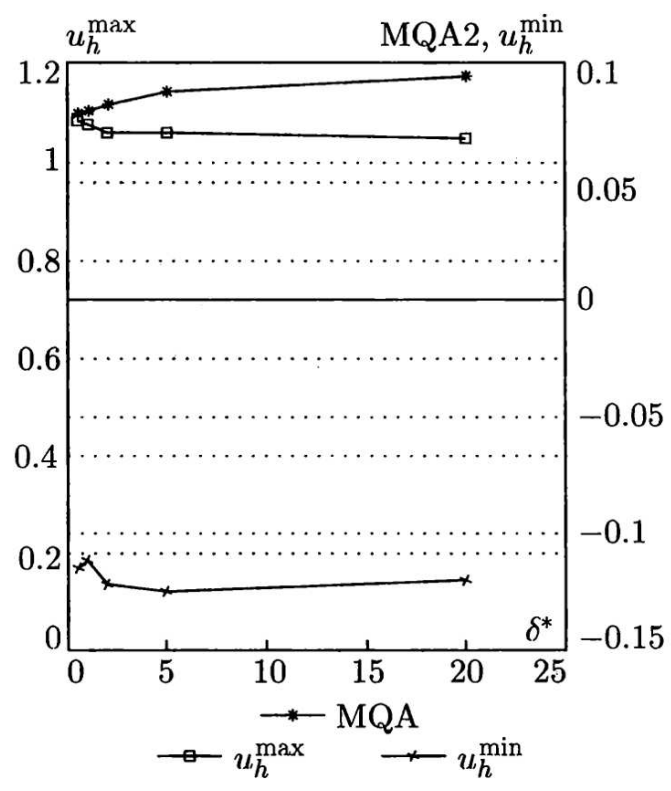

Fig. $2.2 \mathrm{~b}$

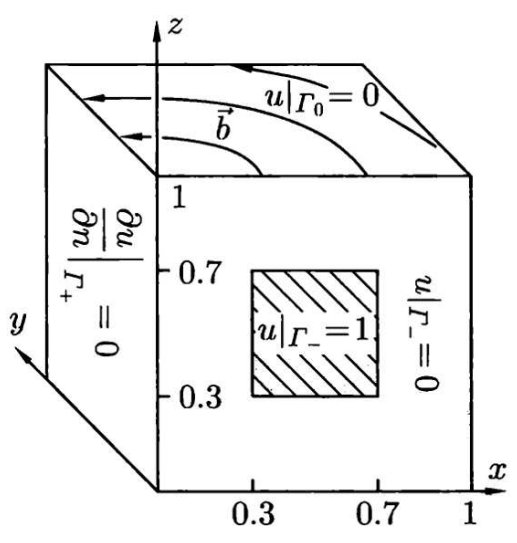

Fig. $2.2 \mathrm{a}$

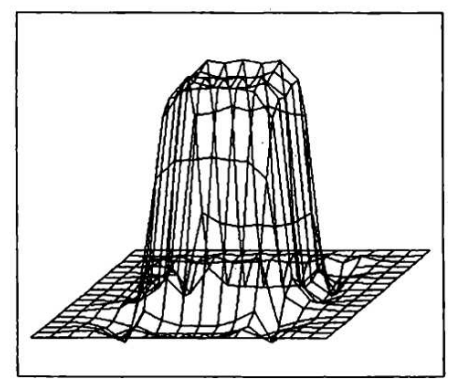

Fig. 2.2c

In the next examples, we discuss the effect of reaction terms in (2.1), (2.2). 
EXAmPle 2.4. Let $\Omega=(0,1)^{2} \subset \mathbb{R}^{2}, \varepsilon=10^{-6}, \mathbf{b}=\left(1-x_{2}^{2}, 0\right)^{\mathrm{T}}, c=5$, $f=0$. At the inflow part of the boundary $\left(x_{1}=0\right)$ let $u=1$; on $\Gamma_{0} \cup \Gamma_{+}$we impose homogeneous Neumann conditions. In the characteristic boundary layer at $x_{2}=0$, there exists a strong interaction of diffusion, convection and reaction terms. The mesh is refined in the neighbourhood of this layer. The SU-solution with $\gamma_{K}=0$ admits a strong discontinuity at $(0,0)$ (cf. Fig. 2.3a with $\delta^{*}=1$ ). The local error at $(0,0)$ is obviously reduced in the GLS-method with $\gamma_{K}=\delta_{K}$ even on a rough mesh (cf. Fig. $2.3 \mathrm{~b}$ with $\delta^{*}=1$ ).

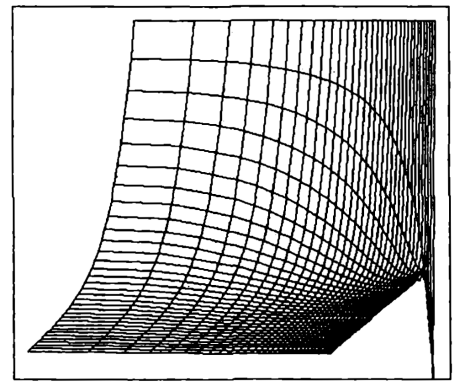

Fig. 2.3a

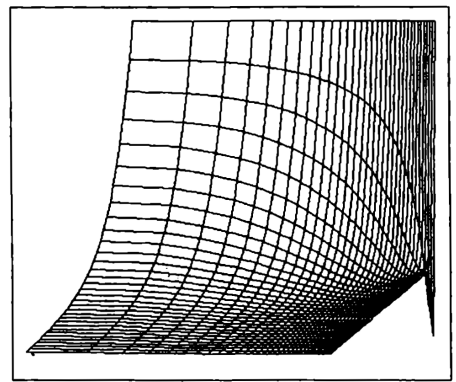

Fig. $2.3 \mathrm{~b}$

EXAMPLE 2.5. Let $\Omega=(0,1)^{2} \subset \mathbb{R}^{2}, \varepsilon=10^{-8}, \mathbf{b}=(0,0)^{\mathrm{T}}, c=f=1$ such that the problem is reaction dominated. Let $u=1$ at $x_{1}=0$ and $x_{2}=0$, but $u=0$ at $x_{1}=1$ and $x_{2}=1$. The solution admits a boundary layer at $x_{2}=1$ and $x_{1}=1$, respectively. The GLS-solution has local oscillations in the neighbourhood of the layers (cf. Fig. 2.4a on a $20 \times 20$-mesh).

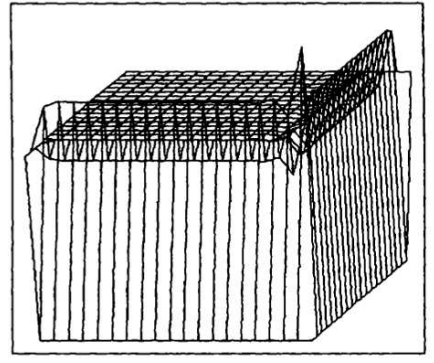

Fig. $2.4 \mathrm{a}$

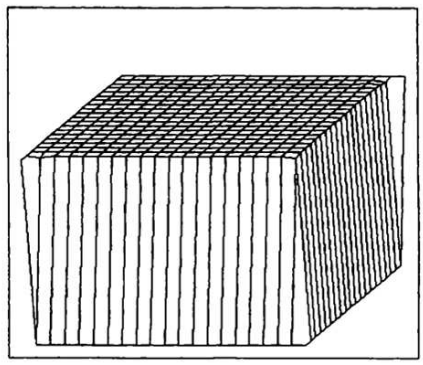

Fig. $2.4 \mathrm{~b}$

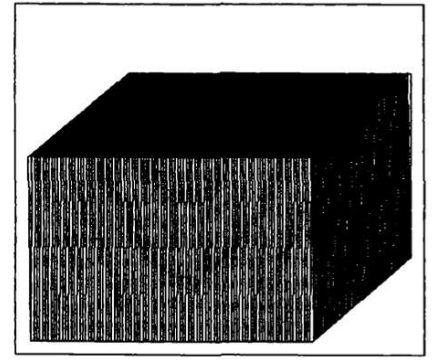

Fig. 2.4c,

[FD] proposes as a remedy the so-called Galerkin-gradient/least-squares method (GGLS):

$$
u_{h} \in V_{h}: \quad B_{S G}\left(u_{h}, v_{h}\right)+\sum_{K} \eta_{K}\left(\nabla\left(L u_{h}-f\right), \nabla\left(L v_{h}\right)\right)_{K}=L_{S G}\left(v_{h}\right) .
$$

For piecewise linear interpolation $(l=1)$, the additional term represents numerical diffusivity according to

$$
\sum_{K} \eta_{K}\left(\nabla\left(c u_{h}-f\right), \nabla\left(c v_{h}\right)\right)_{K}
$$


In Fig. 2.4b and c we give the plot of the GGLS-solution on a $20 \times 20$ - and a $100 \times 100$-mesh, respectively, with $\eta_{K}=0.75 h^{2}, h=h_{K}$.

An error analysis for the GGLS-scheme is given in [FD] in the case of $\mathbf{b}=$ $(0,0)^{\mathrm{T}}$.

\section{Stationary incompressible flow problems.}

3.1. Stabilized Galerkin methods. Let $\Omega \subset \mathbb{R}^{d}, d \leq 3$, be the bounded flow domain with a Lipschitz continuous boundary $\Gamma=\partial \Omega$. We consider the following velocity-pressure formulation of the Navier-Stokes equations governing steady incompressible flow:

$$
\begin{aligned}
N(\mathbf{u}, \widehat{\mathbf{u}}):=-\varepsilon \Delta \mathbf{u}+(\mathbf{u} \cdot \nabla) \mathbf{u}+\nabla p & =\mathbf{f} & & \text { in } \Omega, \\
\nabla \cdot \mathbf{u} & =0 & & \text { in } \Omega
\end{aligned}
$$

where $\widehat{\mathbf{u}}=(\mathbf{u}, p), \mathbf{u}$ and $p$ are velocity and pressure. We assume that for the inverse Reynolds number $\varepsilon$ and a given body force $\mathbf{f}$,

$$
\varepsilon>0, \quad \mathbf{f} \in L^{2}(\Omega)^{d} .
$$

For simplicity we analyze only homogeneous Dirichlet boundary conditions

$$
\mathbf{u}=\mathbf{0} \quad \text { on } \Gamma \text {. }
$$

Let $X:=V \times Q, V:=W_{0}^{1,2}(\Omega)^{d}, Q:=L_{0}^{2}(\Omega):=\left\{q \in L^{2}(\Omega), \int_{\Omega} q d x=0\right\}$. There exists at least one solution $\widehat{\mathbf{u}} \in X$ of (3.1)-(3.3) which is additionally unique for small data

$$
\beta\|\mathbf{f}\|_{V^{*}} \varepsilon^{-2} \leq \omega<1, \quad \beta:=\sup _{\mathbf{u}, \mathbf{v}, \mathbf{w} \in V} \frac{((\mathbf{u} \cdot \nabla) \mathbf{v}, \mathbf{w})_{\Omega}}{|\mathbf{u}|_{V}|\mathbf{v}|_{V}|\mathbf{w}|_{V}} .
$$

Otherwise the solution set of (3.1)-(3.3) is "essentially finite" $[\mathrm{T}]$.

We consider the following simple iteration procedure $(n=0,1, \ldots)$ :

$$
\begin{aligned}
& \widehat{\mathbf{u}}^{n+1} \in X: \quad B_{G}\left(\mathbf{u}^{n}: \widehat{\mathbf{u}}^{n+1}, \mathbf{v}\right)=(\mathbf{f}, \mathbf{v}) \quad \forall \mathbf{v} \in V, \\
& \left(\nabla \cdot \mathbf{u}^{n+1}, q\right)=0 \quad \forall q \in Q
\end{aligned}
$$

for given $\widehat{\mathbf{u}}^{0} \in X$ with

$$
B_{G}(\mathbf{a} ; \widehat{\mathbf{u}}, \mathbf{v}):=\varepsilon(\nabla \mathbf{u}, \nabla \mathbf{v})+\frac{1}{2}\left\{((\mathbf{a} \cdot \nabla) \mathbf{u}, \mathbf{v})_{\Omega}-((\mathbf{a} \cdot \nabla) \mathbf{v}, \mathbf{u})_{\Omega}\right\}-(p, \nabla \cdot \mathbf{v})_{\Omega},
$$

which, according to (3.4), converges for small data to the (unique) solution $\widehat{\mathbf{u}} \in X$ of (3.1)-(3.3) [GR].

In the following we restrict ourselves to stabilized Galerkin schemes for linearized Navier-Stokes problems of type (3.5), (3.6). For a given field a with

$$
\begin{gathered}
\mathbf{a} \in L^{\infty}(\Omega)^{d} \cap H_{\text {div }}(\Omega), \quad \nabla \cdot \mathbf{a}=0 \quad \text { a.e. in } \Omega, \\
H_{\text {div }}(\Omega):=\left\{\mathbf{v} \in L^{2}(\Omega)^{d}, \nabla \cdot \mathbf{v} \in L^{2}(\Omega)\right\}
\end{gathered}
$$

let

$$
\widehat{\mathbf{u}} \in X: \quad B_{G}(\mathbf{a} ; \widehat{\mathbf{u}}, \mathbf{v})=(\mathbf{f}, \mathbf{v})_{\Omega} \quad \forall \mathbf{v} \in V,
$$




$$
(\nabla \cdot \mathbf{u}, q)_{\Omega}=0 \quad \forall q \in Q .
$$

Note that $\mathbf{a} \equiv \mathbf{0}$ corresponds to the Stokes problem.

With a discretization of $\bar{\Omega}$ as introduced in $\S 2$, we define the following conforming finite element interpolation function spaces for velocity and pressure:

$$
\begin{aligned}
X_{h} & :=V_{h} \times Q_{h} \subset X:=V \times Q, \\
V_{h} & :=\left\{\mathbf{v} \in V:\left.\mathbf{v}\right|_{K} \in P_{l}(K)^{d} \forall K \in \mathcal{T}_{h}\right\}, \\
Q_{h} & :=\left\{q \in Q \cap W^{1,2}(\Omega):\left.q\right|_{K} \in P_{k}(K) \forall K \in \mathcal{T}_{h}\right\}
\end{aligned}
$$

with integers $l, k, l \geq 1, k \geq 0$. The basic Galerkin finite element discretization of $(3.8),(3.9)$ reads

$$
\begin{aligned}
\widehat{\mathbf{u}}_{h}=\left(\mathbf{u}_{h}, p_{h}\right) \in X_{h}: & B_{G}\left(\mathbf{a}, \widehat{\mathbf{u}}_{h}, \mathbf{v}_{h}\right)=\left(\mathbf{f}, \mathbf{v}_{h}\right)_{\Omega} & & \forall \mathbf{v}_{h} \in V_{h}, \\
& \left(\nabla \cdot \mathbf{u}_{h}, q_{h}\right)_{\Omega}=0 & & \forall q_{h} \in Q_{h} .
\end{aligned}
$$

Remark 3.1. For discontinuous pressure interpolation in $Q_{h}$, one can introduce in (3.13), (3.14) a jump term

$$
\sum_{K} \beta_{K}\left(\left[p_{h}\right],\left[q_{h}\right]\right) \partial K
$$

where $[p]$ denotes the jump of $p$ across $\partial K$ [DW], [FS].

Numerical oscillations in such mixed methods might be generated by

(a) inappriopriate combinations of velocity/pressure interpolation functions which do not satisfy the inf-sup condition (or Babuška-Brezzi condition)

$$
\inf _{q_{h} \in Q_{h}} \sup _{\mathbf{v}_{h} \in V_{h}} \frac{\left(q_{h}, \nabla \cdot \mathbf{v}_{h}\right)_{\Omega}}{\left\|q_{h}\right\|_{Q}\left|\mathbf{v}_{h}\right|_{V}} \geq \gamma>0
$$

with a mesh-independent constant $\gamma[\mathrm{BF}]$, and/or

(b) the presence of dominant convective terms such that, for the local Reynolds number $\operatorname{Re}_{K}$,

$$
\operatorname{Re}_{K}:=\varepsilon^{-1}\|\mathbf{a}\|_{0, \infty, K} h_{K}>1
$$

As a consequence of (a), simple low order pairs of velocity/pressure interpolation functions (as $\mathrm{P} 1 / \mathrm{P} 1, \mathrm{Q} 1 / \mathrm{Q} 1$ or $\mathrm{Q} 1 / \mathrm{P} 0$ ) which are attractive from the computational point of view (with respect to unsteady 3D flow computations using adaptive mesh refinement and multigrid methods) are not allowed. As a remedy, the following class of stabilized Galerkin methods is considered:

$$
\begin{gathered}
\widehat{\mathbf{u}}_{h}=\left(\mathbf{u}_{h}, p_{h}\right) \in X_{h}: \quad B_{S G}\left(\mathbf{a} ; \widehat{\mathbf{u}}_{h}, \widehat{\mathbf{v}}_{h}\right)=L_{S G}\left(\mathbf{a} ; \widehat{\mathbf{v}}_{h}\right), \\
\left(\nabla \cdot \mathbf{u}_{h}, q_{h}\right)_{\Omega}+\sum_{K} \tau_{K}\left(\nabla \cdot \mathbf{u}_{h}, \nabla \cdot \mathbf{v}_{h}\right)_{K}=0 \quad \forall \widehat{\mathbf{v}}_{h}=\left(v_{h}, q_{h}\right) \in X_{h}
\end{gathered}
$$

with

$$
B_{S G}(\mathbf{a} ; \widehat{\mathbf{u}}, \widehat{\mathbf{v}}):=B_{G}(\mathbf{a} ; \widehat{\mathbf{u}}, \mathbf{v})+\sum_{K}(N(\mathbf{a}, \widehat{\mathbf{u}}), \psi(\mathbf{a}, \widehat{\mathbf{v}}))_{K}
$$




$$
\begin{aligned}
L_{S G}(\mathbf{a} ; \widehat{\mathbf{v}}) & :=(\mathbf{f}, \mathbf{v})_{\Omega}+\sum_{K}(\mathbf{f}, \psi(\mathbf{a}, \widehat{\mathbf{v}}))_{K}, \\
\left.\psi(\mathbf{a}, \widehat{\mathbf{v}})\right|_{K} & :=\delta_{K}(\mathbf{a} \cdot \nabla) \mathbf{v}+\gamma_{K}(-\varepsilon \Delta \mathbf{v}+\nabla q) \quad \forall K \in \mathcal{T}_{h} .
\end{aligned}
$$

Note that a solution of (3.8), (3.9) still satisfies the stabilized formulation (3.17)(3.21). With $\delta_{K}=\gamma_{K}>0$ we recover the GLS method. [Tea] introduced the streamline upwind/pressure stabilizing method with $\delta_{K} \neq \gamma_{K}$ which is similarly defined as the method in Remark 2.4.

3.2. Parameter design and error analysis. For simplicity, we discuss only the GLS-method with $\delta_{K}=\gamma_{K}$; more precisely, we assume the following (minimal) design condition to be satisfied:

(H.4a) $0<\varepsilon \delta_{K} \equiv \varepsilon \gamma_{K}<A_{\delta} h_{K}^{2}, \quad A_{\delta}<\frac{1}{2} C_{I} \quad$ (cf. (2.4)) $\forall K \in \mathcal{T}_{h}$,

$$
\tau_{K}>0 \quad \forall K \in \mathcal{T}_{h}
$$

and continuous pressure interpolation (but cf. Remark 3.1)

$$
Q_{h} \subset Q \cap W^{1,2}(\Omega) .
$$

Let $\|\mid \cdot\|: X_{h}=V_{h} \times Q_{h} \rightarrow \mathbb{R}^{+}$be defined as

$$
\begin{aligned}
\left.\left\|\widehat{\mathbf{u}}_{h}\right\|\right|^{2}:= & \varepsilon\left|\mathbf{u}_{h}\right|_{1,2, \Omega}^{2}+\sum_{K} \tau_{K}\left\|\nabla \cdot \mathbf{u}_{h}\right\|_{0,2, K}^{2} \\
& +\sum_{K} \delta_{K}\left\|-\varepsilon \Delta \mathbf{u}_{h}+(\mathbf{a} \cdot \nabla) \mathbf{u}_{h}+\nabla p_{h}\right\|_{0,2, K}^{2},
\end{aligned}
$$

which is a norm on $X_{h}$ due to (H.5) and $\delta_{K}, \tau_{K}>0$ by (H.4). Furthermore, let with $\delta_{K}=\gamma_{K}$

$$
\widetilde{B}_{G L S}(\mathbf{a} ; \widehat{\mathbf{u}}, \widehat{\mathbf{v}}):=B_{S G}(\mathbf{a} ; \widehat{\mathbf{u}}, \widehat{\mathbf{v}})+(\nabla \cdot \mathbf{u}, q)_{\Omega}+\sum_{K} \tau_{K}(\nabla \cdot \mathbf{u}, \nabla \cdot \mathbf{v})_{K}
$$

such that the GLS-method is rewritten as

$$
\widehat{\mathbf{u}}_{h} \in X_{h}: \quad \widetilde{B}_{G L S}\left(\mathbf{a} ; \widehat{\mathbf{u}}_{h}, \widehat{\mathbf{v}}_{h}\right)=L_{S G}\left(\mathbf{a}, \widehat{\mathbf{v}}_{h}\right) \quad \forall \widehat{\mathbf{v}}_{h} \in X_{h} .
$$

Now we give some auxiliary estimates for the bilinear form $\widetilde{B}_{G L S}(\mathbf{a} ; \cdot, \cdot)$ which follow similarly to Lemmas 2.1, 2.2 in $\S 2$ (for details cf. [LA], Lemma 4.2).

Lemma 3.1. For each $\widehat{\mathbf{v}}_{h}=\left(\mathbf{v}_{h}, q_{h}\right) \in X_{h}, \widetilde{B}_{G L S}\left(\mathbf{a} ; \widehat{\mathbf{v}}_{h}, \widehat{\mathbf{v}}_{h}\right)=\left\|\left|\widehat{\mathbf{v}}_{h}\right|\right\|^{2}$.

Proof. Set $\widehat{\mathbf{v}}_{h}=\widehat{\mathbf{u}}_{h}$ in (3.17)-(3.21) with $\delta_{K}=\gamma_{K}$ and use (3.22).

Lemma 3.2. Under assumptions (H.3), (H.4), (H.5) there exists a positive constant $C$ (independent of $\left.\varepsilon, h_{K}, \delta_{K}, \tau_{K}\right)$ such that $\forall \widehat{\mathbf{v}} \in X_{h}$ and $\forall \widehat{\mathbf{u}} \in X=$ $V \times Q$ with $N(\mathbf{a}, \widehat{\mathbf{u}}) \in L^{2}(\Omega)^{d}$,

$$
\begin{aligned}
\left|\widetilde{B}_{G L S}(\mathbf{a} ; \widehat{\mathbf{u}}, \widehat{\mathbf{v}})\right| \leq & \frac{1}{2}\|\widehat{\mathbf{v}}\| \|^{2}+C\left\{\left.\|\widehat{\mathbf{u}}\|\right|^{2}+\sum_{K} \delta_{K}^{-1}\|\mathbf{u}\|_{0,2, K}^{2}\right. \\
& \left.+\sum_{K} \min \left\{\varepsilon^{-1} ; \tau_{K}^{-1}\right\}\|p\|_{0,2, K}^{2}\right\} . \cdot
\end{aligned}
$$


Using the auxiliary results of Lemmas 3.1 and 3.2, we can now state the following existence and convergence result.

TheOREM 3.3. Under the assumptions (H.3), (H.4), (H.5) there exists a unique solution $\widehat{\mathbf{u}}_{h} \in X_{h}$ of the GLS-method with $\delta_{K}=\gamma_{K}$. Furthermore, if the solution $\widehat{\mathbf{u}}=(\mathbf{u}, p)$ of $(3.8),(3.9)$ satisfies

$$
\mathbf{u} \in V \cap W^{t+1,2}(\Omega)^{d}, \quad 1 \leq t \leq l, \quad p \in Q \cap W^{s+1,2}(\Omega), \quad 0 \leq s \leq k,
$$

then $\widehat{\mathbf{u}}_{h}$ converges to the solution $\widehat{\mathbf{u}}$ of (3.8), (3.9) as follows:

$$
|| \widehat{\mathbf{u}}-\left.\widehat{\mathbf{u}}_{h}\left|\|^{2} \leq C_{1} \sum_{K} h_{K}^{2 t} E_{K}\right| \mathbf{u}\right|_{t+1,2, K} ^{2}+C_{2} \sum_{K} h_{K}^{2 s} F_{K}|p|_{s+1,2, K}^{2}
$$

with constants $C_{1}, C_{2}$ independent of $\varepsilon, h_{K}, \delta_{K}, \tau_{K}$ and

$$
\begin{aligned}
& E_{K}\left(\varepsilon, h_{K}, \delta_{K}, \tau_{K}\right):=\varepsilon+\tau_{K}+\min \left\{\|\mathbf{a}\|_{0, \infty, K}^{2} \delta_{K} ; h_{K}^{2} \delta_{K}^{-1}\right\}, \\
& F_{K}\left(\varepsilon, h_{K}, \delta_{K}, \tau_{K}\right):=\delta_{K}+\min \left\{\varepsilon^{-1} ; \tau_{K}^{-1}\right\} h_{K}^{2} .
\end{aligned}
$$

Proof. The existence and uniqueness of $\widehat{\mathbf{u}}_{h} \in X_{h}$ are a consequence of LaxMilgram's theory and Lemmas 3.1, 3.2.

Let

$$
\begin{aligned}
\widehat{\mathbf{e}}_{h} & \equiv\left(\mathbf{u}_{h}-\mathbf{u}, p_{h}-p\right)=\left(\mathbf{u}_{h}-\pi_{h} \mathbf{u}, p_{h}-\pi_{h} p\right)+\left(\pi_{h} \mathbf{u}-\mathbf{u}, \pi_{h} p-p\right) \\
& \equiv\left(\vartheta_{h}^{u}, \vartheta_{h}^{p}\right)+\left(\eta_{h}^{u}, \eta_{h}^{p}\right) \equiv \widehat{\vartheta}_{h}+\widehat{\eta}_{h} .
\end{aligned}
$$

Then

$$
\begin{aligned}
\left.\left\|\widehat{\vartheta}_{h}\right\|\right|^{2}= & \widetilde{B}_{G L S}\left(\mathbf{a} ; \widehat{\vartheta}_{h}, \widehat{\vartheta}_{h}\right) \\
= & \widetilde{B}_{G L S}\left(\mathbf{a} ; \widehat{\mathbf{e}}_{h}-\widehat{\eta}_{h}, \widehat{\vartheta}_{h}\right)=-\widetilde{B}_{G L S}\left(\mathbf{a} ; \widehat{\eta}_{h}, \widehat{\vartheta}_{h}\right) \\
\leq & \frac{1}{2}\left\|\widehat{\vartheta}_{h} \mid\right\|^{2}+C\left\{\left\|\eta_{h}\right\|\left\|^{2}+\sum_{K} \delta_{K}^{-1}\right\| \eta_{h}^{u} \|_{0,2, K}^{2}\right. \\
& \left.+\sum_{K} \min \left\{\varepsilon^{-1} ; \tau_{K}^{-1}\right\}\left\|\eta_{h}^{p}\right\|_{0,2, K}^{2}\right\} .
\end{aligned}
$$

The triangle inequality and standard interpolation results (cf. (2.23)) imply (3.26)-(3.28).

We now derive an additional design condition for the parameters $\delta_{K}$ and $\tau_{K}$ by balancing the terms in (3.27), (3.28). A simple calculation with the local Reynolds number $\operatorname{Re}_{K}$ yields

$$
\begin{aligned}
& \begin{array}{l}
\delta_{K} \sim h_{K}\left(\|\mathbf{a}\|_{0, \infty, K}\right)^{-1} \\
\tau_{K} \sim h_{K}\|\mathbf{a}\|_{0, \infty, K}
\end{array} \quad \text { if } \operatorname{Re}_{K}=\frac{h_{K}\|\mathbf{a}\|_{0, \infty, K}}{\varepsilon} \geq 1, \\
& \delta_{K} \sim h_{K}^{2} \varepsilon^{-1} \\
& \tau_{K} \sim \varepsilon \\
& \text { if } \operatorname{Re}_{K} \leq 1
\end{aligned}
$$

Then it follows that

$$
E_{K}\left(\varepsilon, h_{K}, \delta_{K}, \tau_{K}\right) \sim \varepsilon+\|\mathbf{a}\|_{0, \infty, K} h_{K} \sim \varepsilon\left(1+\operatorname{Re}_{K}\right),
$$




$$
\begin{aligned}
F_{K}\left(\varepsilon, h_{K}, \delta_{K}, \tau_{K}\right) & \sim \min \left\{\frac{h_{K}}{\|\mathbf{a}\|_{0, \infty, K}} ; \frac{h_{K}^{2}}{\varepsilon}\right\} \\
& \sim \frac{h_{K}}{\|\mathbf{a}\|_{0, \infty, K}} \min \left\{1 ; \operatorname{Re}_{K}\right\}
\end{aligned}
$$

and

COROLlary 3.4. Under the assumptions (H.3), (H.4*), (H.5), the following error estimate for the GLS-method with $\delta_{K}=\gamma_{K}$ is valid:

$$
\begin{aligned}
\left.\left\|\widehat{\mathbf{u}}-\widehat{\mathbf{u}}_{h}\right\|\right|^{2}:= & \varepsilon\left|\mathbf{u}-\mathbf{u}_{h}\right|_{1,2, \Omega}^{2}+\sum_{K} \tau_{K}\left\|\nabla \cdot\left(\mathbf{u}-\mathbf{u}_{h}\right)\right\|_{0,2, K}^{2} \\
& +\sum_{K} \delta_{K}\left\|N\left(\mathbf{a}, \widehat{\mathbf{u}}-\widehat{\mathbf{u}}_{h}\right)\right\|_{0,2, K}^{2} \\
\leq & C_{1} \sum_{K} h_{K}^{2 t} \varepsilon\left(1+\operatorname{Re}_{K}\right)|\mathbf{u}|_{t+1,2, K}^{2} \\
& +C_{2} \sum_{K} h_{K}^{2 s+1}\left(\|\mathbf{a}\|_{0, \infty, K}\right)^{-1} \min \left\{1 ; \operatorname{Re}_{K}\right\}|p|_{s+1,2, K}^{2} \cdot
\end{aligned}
$$

We conclude with some remarks.

Re mark 3.2. The estimate (3.31) involves control of weighted discrete residuals of the GLS-method according to

$$
\begin{aligned}
& \varepsilon \sum_{K} \min \left\{1 ; \operatorname{Re}_{K}\right\}\left\|\nabla \cdot \mathbf{u}_{h}\right\|_{0,2, K}^{2} \\
&+\sum_{K} h_{K}\left(\|\mathbf{a}\|_{0, \infty, K}\right)^{-1} \min \left\{1 ; \operatorname{Re}_{K}\right\}\left\|N\left(\mathbf{a}, \widehat{\mathbf{u}}_{h}\right)-\mathbf{f}\right\|_{0,2, K}^{2} \\
& \leq \text { r. h. s. of }(3.31),
\end{aligned}
$$

which could be exploited in adaptive mesh refinement methods (cf. [Jb]).

Remark 3.3. For Stokesian flow $(\mathbf{a} \equiv \mathbf{0})$ we recover essentially the result of $\left[\mathrm{DW}\right.$. Note that $\operatorname{Re}_{K}=0$.

Remark 3.4. It is possible to repeat the analysis of the GLS-method for the original Navier-Stokes problem (3.1)-(3.3) replacing a with $\mathbf{u}_{h}$. Then Theorem 3.3 and Corollary 3.4 remain valid with $\mathbf{a}=\mathbf{u}_{h}$ in the small data case (3.4). Furthermore, an asymptotic error estimate holds for branches of nonsingular solutions of (3.1)-(3.3) (cf. [GR], [LA]).

3.3. Numerical results. We present simple $2 \mathrm{D}$ examples with Lagrangian $\mathrm{P} 1 / \mathrm{P} 1$ interpolation $(l=k=1)$ of velocity and pressure which do not satisfy the inf-sup condition (3.15).

EXAmple 3.1. As an accuracy test we performed calculations for Stokes flow problems $(\mathbf{a} \equiv \mathbf{0})$ for the fully developed Poiseuille flow with Dirichlet outlet (a) 
and with free outlet (b) and for two body force problems with $\mathbf{f} \not \equiv \mathbf{0}$ (c, d cf. $[\mathrm{P}])$. Averaged discrete numerical convergence rates are given in Table 3.1. $\Omega_{h}$ denotes the set of finite element nodes in $\bar{\Omega}$.

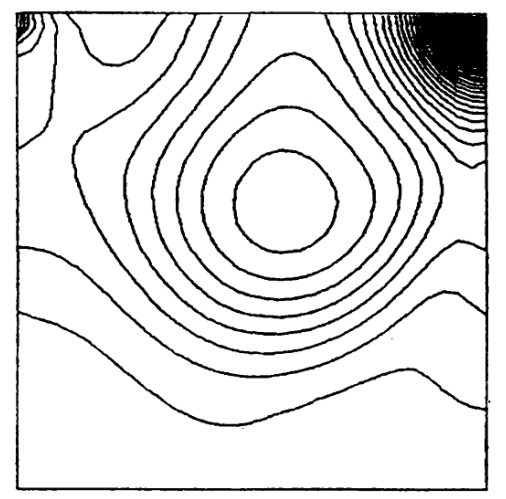

Fig. 3.1a

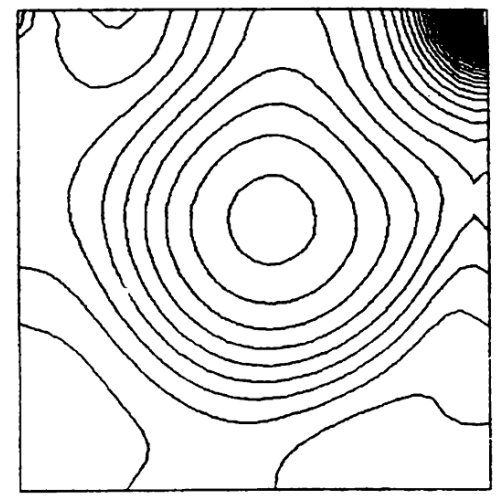

Fig. 3.2a

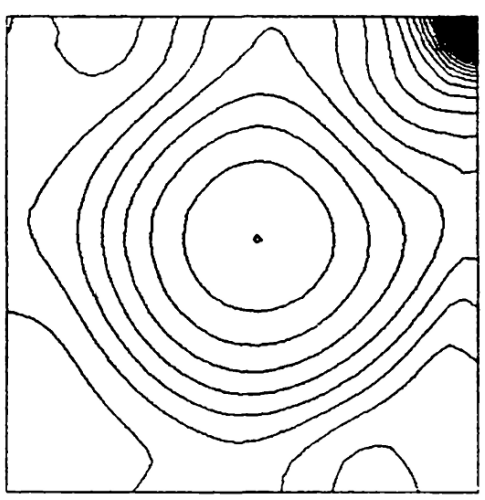

Fig. 3.3a

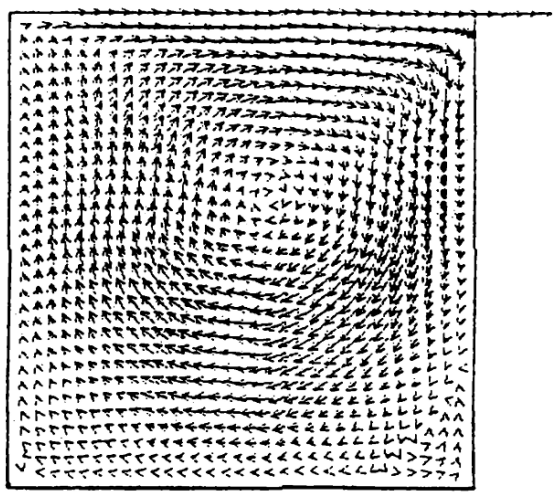

Fig. 3.1b

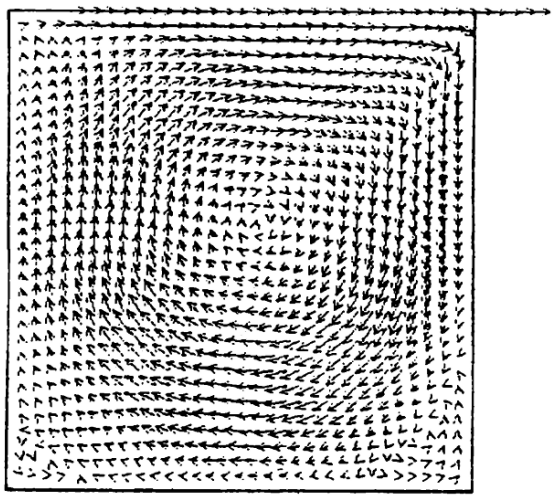

Fig. $3.2 b$

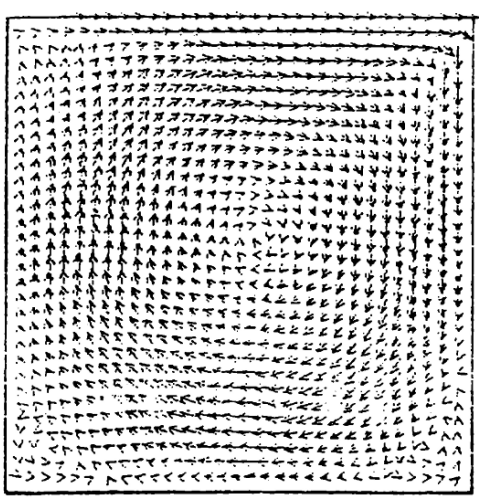

Fig. $3.3 \mathrm{~b}$ 
Table 3.1. Discrete numerical convergence rates (averaged) for Stokesian flow problems

\begin{tabular}{|c|c|c|c|c|}
\hline example & a & b & c & d \\
\hline$\left\|\mathbf{u}-\mathbf{u}_{h}\right\|_{0,2, \Omega_{h}}$ & 1.88 & 1.82 & 1.41 & 1.77 \\
$\left\|\mathbf{u}-\mathbf{u}_{h}\right\|_{0, \infty, \Omega_{h}}$ & 1.60 & 1.85 & 1.57 & 1.94 \\
$\left\|p-p_{h}\right\|_{0,2, \Omega_{h}}$ & 1.89 & 1.64 & 1.42 & 1.75 \\
$\left\|p-p_{h}\right\|_{0, \infty, \Omega_{h}}$ & 1.11 & 1.09 & 1.08 & 1.21 \\
$\left\|\nabla \cdot \mathbf{u}_{h}\right\|_{0, \infty, \Omega_{h}}$ & 1.00 & 0.99 & 0.99 & 0.89 \\
\hline
\end{tabular}

EXAMPLE 3.2. As an example for the Navier-Stokes problem we considered the standard driven cavity square problem with $\mathbf{f} \equiv \mathbf{0}$. The velocity is prescribed to zero at the lower, left and right parts of the boundary and to $(1,0)$ on the upper part of the boundary. In Fig. 3.1 to 3.3 we present the results for Reynolds numbers 400, 1000 and 3000 on an equidistant $32 \times 32$-mesh after 100 iteration steps of (3.5), (3.6). The results for Re $=400$ and 1000 are comparable with those given in [Tea], [Teb] and [HS].

\section{References}

[BF] F. Brezzi and M. Fortin, Mixed and Hybrid Finite Element Methods, Springer, New York 1991.

[DW] J. Douglas, Jr., and J. Wang, An absolutely stabilized finite element method for the Stokes problem, Math. Comp. 52 (186) (1989), 495-508.

[FD] L. P. Franca and E. G. Dutra do Carmo, The Galerkin-gradient-least-squares method, Comput. Methods Appl. Mech. Engrg. 74 (1989), 41-54.

[FFH] L. P. Franca, S. L. Frey and T. J. R. Hughes, Stabilized finite element methods: I. Applications to the advective-diffusive model, ibid., to appear.

[FS] L. P. Franca and R. Stenberg, Error analysis of some Galerkin-least-squares methods for the elasticity equations, SIAM J. Numer. Anal. (1991), to appear.

[GR] V. Girault and P. A. Raviart, Finite Element Methods for Navier-Stokes Equations. Theory and Algorithms, Springer, 1986.

[HS] P. Hansbo and A. Szepessy, A velocity-pressure streamline diffusion finite element method for the incompressible Navier-Stokes equations, Comput. Methods Appl. Mech. Engrg. 84 (1990), 175-192.

[Hu] T. J. R. Hughes, Recent progress in the development and understanding of SUPG methods with special reference to the compressible Euler and Navier-Stokes equations, Internat. J. Numer. Methods Fluids 7 (1987), 1261-1275.

[Ja] C. Johnson, Numerical Solution of Partial Differential Equations by the Finite Element Method, Studentlitteratur, Sweden, 1987.

[Jb] —, Adaptive finite element methods for diffusive and convective problems, Comput. Methods Appl. Mech. Engrg. 82 (1990), 301-322.

[KR] R. Kornhuber and R. Roitzsch, On adaptive grid refinement in the presence of internal and boundary layers, IMPACT of Computing in Science and Engineering 2 (1990), 40-72.

[LA] G. Lube and A. Auge, Regularized mixed finite element approximations of incompressible flow problems. II. Navier-Stokes flow, preprint, TU Magdeburg, 1991. 
[P] R. Pierre, Simple $C^{0}$ approximation for the computation of incompressible flow, Comput. Methods Appl. Mech. Engrg. 68 (1988), 205-227.

[T] R. Temam, Navier-Stokes Equations and Nonlinear Functional Analysis, CBMS-NSF Regional Conf. Ser. in Appl. Math. 41, SIAM, 1983.

[Tea] T. E. Tezduyar, R. Shih, S. Mittal and S. E. Ray, Incompressible flow computations with stabilized bilinear and linear equal-order interpolation velocity-pressure elements, preprint UMSI 90/165, Univ. of Minnesota, 1990.

[Teb] T. E. Tezduyar, Stabilized finite element formulations for incompressible flow computations, von Karman Institute for Fluid Dynamics, Lecture Series 1991-01.

[Wa] L. B. Wahlbin, Local behavior in finite element methods, in: Handbook of Numerical Analysis, P. G. Ciarlet and J. L. Lions (eds.), Vol. II, North-Holland, 1990.

[We] D. Weiß, Numerische Simulation von Temperaturfeldern bei Schweißvorgängen, Forschungsbericht, TU Magdeburg, 1991. 